\title{
Management of temporomandibular joint disorders
}

\author{
Louis G. Mercuri*
}

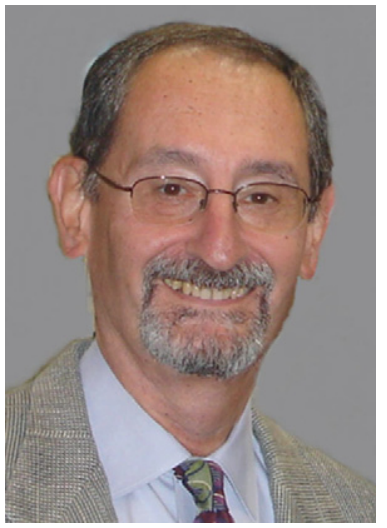

Orthopedics is the branch of medicine/surgery concerned with acute, chronic, traumatic, overuse injuries and other disorders of the musculoskeletal system. The temporomandibular joint (TMJ) is affected by all the same traumatic injuries and pathologic diseases as is any other body joint. Therefore, as is true in those joints, disorders the TMJ and associated structures should be managed as a "composite unit" consisting of not of just its articulating bones, disc and cartilage, but also all of the components that give rise to its form and allow it to function. Touting the TMJ completely different than all other body joints is not only functionally irrational but also anatomically naïve.

The new paradigms determined and validated for pain associated with muscle, ${ }^{1}$ neuroplasticity and central sensitization, $^{2}$ and the genomics of pain ${ }^{3}$ all speak to the importance of addressing all of the anatomical and functional complexities of the TMJ within any management scheme.

Most diseases in medicine are managed, not cured think of diabetes, hypertension, etc. Unfortunately, our dental education often teaches us that dental-related diseases are curable (i.e., if there is decay and it is removed from the tooth and replaced with a restoration, the tooth is cured. The same holds true for extraction of a non-restorable or periodontally involved tooth... on and on).

The "cure" model can lead dentally trained individuals to accept as fact that if temporomandibular joint disorders (TMD) are all mechanical and "dentally related," these too can be "cured." This false impression frequently results in irreversible, potentially harmful and often unscientific "treatment" options. The numerous signs and symptoms commonly attributed to TMD challenge any practitioner to "cure" them all! Therefore the operative word in TMD must be "management" not "treatment."

The TMJ articulation obeys all of the same physical and anatomical kinematic laws as all other joints in the body despite its position within the anatomy. The misconception that occlusion as the cause of TMD was based on the presence of contorted ectodermal structures (teeth) relationships resulting in TMD has been disproven. ${ }^{4}$

Based on imaging studies of normal TMJs, it is now well established that the TMJ disc's function is more important than its position. Internal derangement does not result in progressive TMJ deterioration as was once decreed..$^{5-7}$

The misguided concepts of the past often resulted in the opinion that "... treatment is necessary to prevent progression of internal derangement disorders from progressing to degenerative joint disease". ${ }^{8}$ But in truth, for years TMD has been characterized as self-limiting, or non-progressive conditions. ${ }^{9}$ We now know that over time, without any "treatment," patients with TMD even "get better". 10,11

Further, if TMD is truly a chronic, progressive, non-selflimiting condition, one would expect that its incidence in the population would increase with age. However, the data clearly reveals that $12 \%$ overall prevalence of TMD pain that peaked in women in the childbearing years fell to $<4 \%$ for women 65 years of age and older; even lower for men. ${ }^{12}$

So why is there even a controversy? Mohl and Ohrbach ${ }^{13}$ provide some insight. They suggest the following: (1) insufficient objective scientifically derived clinical evidence - no good Random Clinical Trials; (2) failure to effectively communicate existing evidence to the profession - practitioners don't read the literature; (3) failure to use wellcommunicated scientific evidence - if we read it, we must use it; (4) dependence on clinical trial and error instead of the scientific method; (5) over-dependence on subjective reports of clinical success - too anxious for the magic bullet; and (6) low appreciation that clinical successes, though noteworthy, are not scientific proof of cause and effect. 
The experience of the past 150 years in the diagnosis and management of chronic orofacial pain conditions has shown that a mechanistic, narrow approach (e.g., occlusion, TMJ disc position) is likely to produce iatrogenic harm (i.e., unnecessary equilibrations, extractions, restorations, TMJ surgery, etc). ${ }^{2}$

TMD "tunnel vision" can lead to misdiagnosis of potentially more serious problems that mimic either TMD pain distribution or limitation of jaw opening possibly resulting in unnecessary "treatments" or other serious misdiagnosis consequences for the patient.

The lesson to be learned is that unless practitioners and investigators subscribe to an orthopedic philosophy of TMD management rather than the mechanistic "cure" approaches of the past, TMD will continue to be a mystery in the future.

\section{REFERENCES}

1. Murray GM, Peck CC. Orofacial pain and jaw muscle activity: a new model. J Orofac Pain. 2007;21:263-278.

2. Turp JC, Hugger A, Sommer C, eds. The Puzzle of Orofacial Pain: Integrated Research into Clinical Management. Basel: Karger; 2007.

3. Tasaki MM, Westesson PL, Isberg AM, et al. Classification and prevalence of TMJ disc displacement in patients and asymptomatic volunteers. Am J Orthod Dentofacial Orthop. 1996;109:249-262.

4. Stohler C. Management of dental occlusion. In: Laskin DM, et al., eds. TMDs An Evidence-Based Approach to Diagnosis and Treatment. Chicago: Quintessence; 2006:403-411.

5. Katzberg RW, Westesson PL, Tallents RH, et al. Anatomic disorders of the TMJ disc in asymptomatic subjects. J Oral Maxillofac Surg. 1996;54:147-153.

6. Larheim TA, Westesson PL, Sano T. Temporomandibular joint disc displacement: comparison in asymptomatic volunteers and patients. Radiology. 2001;218:428-432.
7. Schmitter M, Rammelsberg P, Hassel A, et al. Evaluation of disc position and prevalence of internal derangement in a sample of the elderly by gadolinium-enhanced magnetic resonance imaging. Oral Surg Oral Med Oral Path Oral Radiol Endod. 2008;106:872-878.

8. Rasmussen OC. Description of population and progress of symptoms in a longitudinal study of temporomandibular joint arthropathy. Scand J Dent Res. 1981;89:196-203.

9. Rugh JD, Solberg WK. Psychological implications in temporomandibular joint dysfunction. Oral Sci Rev. 1976;7:3-30.

10. Bertolami CN, Gay T, Clark GT, et al. Use of sodium hyaluronate in treating temporomandibular joint disorders: a randomized, double-blind, placebo-controlled clinical trial. J Oral Maxillofac Surg. 1993;51:232-242.

11. Kurita K, Westesson PL, Yuasa H, et al. Natural course of untreated symptomatic temporomandibular joint disc displacement without reduction. J Dent Res. 1998;77: $361-365$.

12. Van Korff M, Dworkin SF, LeResche L, et al. An epidemiologic comparison of pain complaints. Pain. 1988;32: 173-183.

13. Mohl ND, Ohrbach R. The dilemma of scientific knowledge versus clinical management of TMD. J Prosthet Dent. 1992;67:113-120.

Louis G. Mercuri, DDS, MS, Retired Professor of Surgery, Loyola University Chicago Medical Center, Ex Member, Oral and Maxillofacial Surgery faculty, University of Illinois Chicago College Of Dentistry \& Virginia Commonwealth University Medical College of Virginia School of Dentistry, Richmond, Virginia. Ex Chairman, Department of Oral and Maxillofacial Surgery, Chicago Michael Reese Hospital and Medical Center, University of Illinois Chicago College of Dentistry \& Loyola University Chicago Medical Center. Clinical Consultant for TMJ Concepts, Ventura California. Fellow of the American Association of Oral and Maxillofacial Surgeons. Retired Diplomate of the American Board of Oral and Maxillofacial Surgeons. Email: lgm@tmjconcepts.com. 